\title{
Pequenas empresas industriais de longa permanência no mercado: uma análise a partir da literatura e de evidências empíricas
}

\author{
Long stay of small industrial firms in the market: an analysis \\ from the literature and empirical evidence
}

\author{
Maria Carolina de Azevedo Ferreira de Souza ${ }^{1}$ \\ Leonel Mazzali² \\ Rodrigo Lanna Franco da Silveira ${ }^{1}$ \\ Miguel Juan Bacic ${ }^{1}$
}

\begin{abstract}
Resumo: O trabalho visa identificar a influência da idade, tamanho, escopo e efeitos do local sobre a probabilidade de sobrevivência a longo prazo das pequenas empresas industriais. É um estudo descritivo que se apoia em um banco de dados com informações das pequenas empresas industriais da região de Campinas, construído a partir do Cadastro Industrial da Região de Campinas e dados da Secretaria da Fazenda do Estado de São Paulo (SEFAZ). O estudo evidenciou que a amplitude da linha de produtos, a abrangência em termos de mercado e o tamanho exercem influência sobre a probabilidade de sobrevivência das pequenas empresas industriais.
\end{abstract}

Palavras-chave: Pequenas empresas. Longevidade. Gestão estratégica.

\begin{abstract}
This study aims to identify the influence of age, size, scope, and location on the probability of long-term survival of small industrial enterprises. This is a descriptive study based on a database that embodies information of small business located in the region of Campinas, São Paulo state, with data from the Campinas Industrial Region and São Paulo State Treasury Office. The present study showed that product line breadth, market coverage, and size influence the probability of survival of small industrial enterprises.
\end{abstract}

Keywords: Small businesses. Longevity. Strategic management.

\section{Introdução}

A crescente importância das pequenas empresas (PEs), pela significativa participação no total de empresas existentes e no total de empregos gerados, tem estimulado uma série de estudos enfatizando a sua funcionalidade nas estruturas produtivas e a sua contribuição para o emprego. Uma das questões que geralmente permeiam esses estudos refere-se à continuidade delas no mercado, apesar das desvantagens que dificultam o alcance de competitividade (AUDRETSCH et al., 2009; HEADD; KIRCHHOFF, 2007; NAJBERG; PUGA; OLIVEIRA, 2002).

Existe uma ampla discussão sobre os fatores que podem explicar a mortalidade dos empreendimentos de pequeno porte e sobre aqueles que favorecem a sobrevivência, após a sua constituição. Entretanto, ainda há poucos estudos com ênfase na busca de elementos que contribuam para explicar a continuidade de pequenas empresas no mercado por muitos anos, superando a fase de maior risco de mortalidade. Diante dessa constatação, surge a questão: apesar de todas as restrições e fragilidades e a despeito das profundas modificações no ambiente macroeconômico, como explicar a presença de pequenas empresas de longa permanência no mercado?

Ao se pensar nas causas de sobrevivência e de mortalidade de empresas, as características estruturais do setor em que concorrem, particularmente quanto ao seu tamanho, seu poder de mercado, sua natureza, a intensidade da concorrência e sua forma de inserção devem ser levadas em conta. Independentemente da estrutura do setor e das formas de inserção, fatores relacionados às características dos gestores e às suas escolhas estratégicas, incluindo as relativas à localização, ao escopo quanto aos produtos e à abrangência geográfica de atuação, são igualmente

\footnotetext{
${ }^{1}$ Departamento de Teoria Econômica, Universidade Estadual de Campinas - Unicamp, CEP 13083-847, Campinas, SP, Brasil, e-mail: crln.sz@gmail.com; rodrigolanna@eco.unicamp.br; bacic@eco.unicamp.br

${ }^{2}$ Programa de Mestrado em Administração, Faculdades Metropolitanas Unidas - FMU, CEP 01508-010, São Paulo, SP, Brasil, e-mail: leonel_mazzali@uol.com.br
} 
importantes para o desempenho da empresa. Os resultados obtidos até determinado momento, por sua vez, explicam em grande medida a continuidade (tempo de vida) e o tamanho da empresa, tendo efeito importante sobre o risco de mortalidade.

De acordo com Le Mens, Hanann e Pólos (2011), os resultados passados ("herança" positiva ou negativa) têm mais peso sobre o risco de mortalidade da empresa em determinado momento do que seu desempenho naquele particular instante. Essa proposição implica que na análise da relação idade e risco de mortalidade das empresas seja considerada não só a dotação inicial de capital organizacional (recursos financeiros e não financeiros) mas, principalmente, a evolução temporal do desempenho, do fluxo líquido de recursos. Essa evolução depende, em grande medida, do aprendizado pela experiência acumulada. Portanto, uma relação positiva, ou negativa, entre risco de mortalidade e idade da empresa vai depender das decisões dos gestores e do desempenho da empresa nos anos iniciais. Desempenho negativo levará a uma erosão da dotação inicial. Desempenho positivo levará à acumulação, ocorrendo o crescimento da dotação inicial de recursos e do capital organizacional da empresa.

Sem desconsiderar a importância de fatores externos, a análise neste trabalho sobre a longevidade de pequenas empresas privilegia a evolução temporal do desempenho organizacional, refletindo a capacidade de identificação e aproveitamento de recursos e a eficácia da empresa em conquistar e assegurar posições no mercado.

Tendo por referência as contribuições de Burgelman (1991) e Burgelman e Grove (2007), a evolução temporal do desempenho organizacional está, em essência, associada à combinação de duas trajetórias (ciclos) de desenvolvimento. A primeira, denominada pelos autores de "indução", está atrelada a um processo de gestão estratégica nucleado no aproveitamento das oportunidades existentes. No entanto, a longevidade da empresa depende de sua capacidade não só de gerar rentabilidade nas atividades atuais mas também de entrar em novos negócios. Tendo em conta mudanças no ambiente estratégico, é essencial que a empresa ingresse em uma segunda trajetória, denominada pelos autores de "autônoma", por estar fora do escopo da estratégia corrente.

Para o entendimento da sobrevivência das organizações é necessário identificar os fatores subjacentes aos dois processos estratégicos, de indução e autônomo, e avaliar sua influência relativa. A literatura sobre a teoria da firma pode contribuir nesse sentido. Levando em conta a combinação de algumas das principais abordagens (ecologia das organizações, teoria evolucionista da empresa, visão da empresa baseada em recursos e aprendizagem organizacional), é possível selecionar quatro principais fatores: tempo de vida; tamanho (volume dos recursos e das operações); escopo das atividades (amplitude da linha de produtos e abrangência em termos dos mercados); e efeitos do local no qual a empresa está instalada (economia de localização).

Este trabalho visa, como objetivo principal, contribuir para o entendimento desses fatores, buscando identificar o que os influencia e, principalmente, identificar a influência desse conjunto de fatores (idade, tamanho, escopo e efeitos do local) sobre a probabilidade de sobrevivência a longo prazo das pequenas empresas industriais. Mais precisamente: busca-se avaliar se a posição da empresa quanto ao nível desses quatro fatores em determinado ano base $(t)$, correspondendo a 1975 (no caso deste estudo), de alguma forma influencia a probabilidade de sobrevivência das pequenas empresas no tempo $(t+n)$, correspondendo a 2007 (no caso deste estudo).

O trabalho está estruturado em cinco seções, além desta primeira dedicada à introdução. Na segunda seção são apresentados e discutidos os conceitos de capital organizacional e evolução temporal do desempenho da organização e sua relação com a sobrevivência. A terceira aprofunda a discussão sobre os fatores subjacentes à evolução temporal do desempenho da organização. A quarta é dedicada aos procedimentos metodológicos. A quinta apresenta e discute os resultados da pesquisa empreendida. Finalmente, na sexta seção são apresentadas as conclusões e indicadas possibilidades para pesquisas futuras.

\section{Capital organizacional, evolução temporal do desempenho e sobrevivência da organização}

Utilizando como variável nuclear o estoque de capital organizacional, Levinthal (1991) explora as implicações da evolução temporal do desempenho sobre o padrão de mortalidade das organizações. De forma semelhante ao enfoque da empresa baseada em recursos (WERNERFELT, 1984; RUMELT, 1984), o autor concebe capital organizacional como uma variedade de ativos acumulados pela empresa ao longo do tempo. O capital organizacional abrange ativos financeiros e não financeiros. No âmbito dos não financeiros, destacam-se: consolidação de laços com agentes externos à organização (fornecedores, clientes e instituições financeiras), desembocando na formação/inserção em redes de distribuição e de fornecimento; comprometimento e motivação por parte dos agentes internos à organização, que se traduz na conformação de um sistema de papéis e regras que direcionam o comportamento e a decisão dos indivíduos; e formação e consolidação de habilidades (competências) técnicas e sociais essenciais para a operação do negócio. 
O ponto central da argumentação é que o risco de mortalidade de uma organização depende mais do seu estoque de recursos (financeiros e não financeiros) do que de seus resultados atuais. O estoque de capital organizacional atuaria como um amortecedor dos efeitos de resultados insatisfatórios em determinados períodos. Entretanto, sublinha o autor, essa barreira contra os efeitos temporários adversos tem um limite inferior, um nível crítico, de modo que se o desempenho organizacional for sistematicamente insuficiente, o estoque de recursos se reduz e, por consequência, o manto protetor deixa de existir. $\mathrm{Na}$ análise da continuidade (sobrevivência) da organização devem ser especificadas as condições iniciais, ou, mais precisamente, o que constitui a empresa no momento de sua fundação; e as modificações no estoque de capital organizacional, com evolução temporal do desempenho da organização ao longo do tempo.

Ao tratar da evolução temporal do estoque de recursos, Levinthal (1991) visualiza as estratégias como meios de governar a evolução da empresa. Apoiando-se em Winter (1987), o autor entende as mudanças ao longo do tempo, no nível do capital organizacional, como resultado não só do comportamento sistemático do desempenho organizacional, intimamente ligado à qualidade do processo decisório, mas também do efeito de fatores aleatórios.

No momento da fundação, o capital organizacional equivale às dotações iniciais de recursos. Com o tempo, como apontam Le Mens, Hanann e Pólos (2011), a dotação inicial sofrerá depreciação, mas o capital organizacional poderá ser recomposto e mesmo ampliado se a organização for capaz de extrair (captar) novos recursos do seu ambiente relevante. A capacidade de extrair recursos do ambiente relevante está associada ao componente sistemático do desempenho organizacional, refletindo a eficácia da empresa para atrair e manter posições no mercado. Significa dizer que o desempenho relevante é o relativo, em comparação com os concorrentes, e não o absoluto. O capital organizacional é ampliado a partir da busca pelo "desempenho superior". Conforme destacado por Burgelman (1991) e Burgelman e Grove (2007), a capacidade de a empresa obter e manter posições competitivas está associada à combinação de duas trajetórias (ciclos) de desenvolvimento.

A primeira trajetória diz respeito a um processo estratégico induzido, ou por indução, no qual as "regras do jogo" não se alteram (implícita ou explicitamente) por longos períodos de tempo, engendrando uma forte tendência de inércia estratégica entre os integrantes da indústria. O processo estratégico por indução se apoia no aprofundamento da exploração das oportunidades existentes; reflete a busca constante pelo atendimento eficiente e eficaz de uma demanda já conhecida. Tem como base o refinamento do conhecimento da empresa, acumulado a partir da aprendizagem pela experiência em um ambiente com o qual as empresas estão familiarizadas e que pode ser claramente delineado ao se definir a estratégia competitiva. Tal situação é de "estabilidade", de "conforto", mas não suficiente para um caminho de expansão.

O caráter mutável do ambiente e a intensificação da concorrência exigem que a empresa seja capaz de adentrar e percorrer uma segunda trajetória, denominada pelos autores de "autônoma", com relação ao conhecimento atual da empresa. No núcleo dessa estratégia está um processo de gestão estratégica fundamentado na percepção e aproveitamento de novas oportunidades, como já havia sido proposto por Penrose (2006). Em contraste com a primeira trajetória, esta se apoia na variação, na experimentação, na flexibilidade, na inovação.

Burgelman e Grove (2007) chamam a atenção para o elevado risco inerente à estratégia autônoma relativamente à estratégia corrente (por indução). Esse processo envolve custos significativos, exige recursos que podem comprometer o funcionamento e a manutenção das posições atuais. Nesse sentido, concluem que as empresas bem-sucedidas por longos períodos de tempo são aquelas capazes de superar as dificuldades da manutenção do equilíbrio entre os dois processos estratégicos (o que só pode ser constatado a posteriori).

As propostas de Burgelman e Grove (2007) abrem espaço para a organização da literatura relevante sobre a longevidade das empresas em torno da identificação dos fatores subjacentes aos dois processos estratégicos, o que será objeto da seção seguinte.

\section{Fatores subjacentes à evolução temporal do desempenho da organização}

Ao lado de aspectos ligados ao ambiente macroeconômico e ao ambiente concorrencial, os principais fatores apontados pelas pesquisas como determinantes da mortalidade nos anos iniciais dos empreendimentos têm relação com a falta de capacidade empresarial; inexperiência do empreendedor; falta de plano de negócios ou plano mal formulado; aporte de capital próprio inferior às reais necessidades do empreendimento; e desconhecimento de aspectos básicos relacionados às operações e à gestão. Essas causas não são excludentes, antes se somam e ampliam o conjunto de fatores que contribuem para a mortalidade das empresas em seus estágios iniciais.

No tocante à sobrevivência, devem-se considerar características estruturais, tais como reduzida economia de escala, fortes desvantagens quanto ao acesso a fontes de financiamento, baixo poder de negociação com clientes e fornecedores e dificuldades de articulação com outras empresas. Enfim, o 
conjunto de assimetrias que as desfavorecem define e delimita o pequeno negócio, representando forte desvantagem relativamente às organizações com maior disponibilidade de capital. Características estruturais dizem respeito, na linguagem da literatura sobre sobrevivência das organizações, de um lado, aos atributos da indústria e do ambiente concorrencial em geral e, de outro lado, aos "atributos imanentes à empresa", de modo particular a idade e o tamanho. Thornhill e Amit (2003) analisaram diversos estudos empíricos desenvolvidos nos EUA e na Inglaterra, em vários setores, no período compreendido entre 1970 e 2000, e constataram que tais características ocupam espaço importante nesses estudos.

No âmbito da indústria e do mercado, o tamanho e a taxa de crescimento da demanda são considerados importantes determinantes da sobrevivência (VAN WISSEN, 2002). Consideram-se ainda os efeitos da fase do ciclo do produto na indústria. Nos estágios iniciais, aumenta a entrada de novas empresas com base na inovação de produto. No entanto, à medida que a indústria amadurece, a inovação de processo se torna dominante e as economias de escala passam a representar forte vantagem competitiva, elevando a taxa de mortalidade das empresas (principalmente daquelas que não conseguem obter economia de escala). Em decorrência, a indústria tende a se tornar mais concentrada, com menos espaço para as pequenas empresas.

Ainda no que se refere às características da indústria, esses estudos levam em conta a dependência da densidade, que diz respeito ao impacto do número total de empresas no interior da indústria sobre a taxa de mortalidade. Carroll e Hannan (2000) oferecem uma síntese dos resultados das pesquisas empreendidas nessa linha. Tais resultados indicam dois efeitos em sentido oposto: o crescimento da densidade populacional aumenta tanto a legitimação, favorecendo a sobrevivência, quanto torna mais intensa a competição (rivalidade), que tem impacto negativo na sobrevivência das empresas.

No âmbito dos atributos inerentes à empresa, tempo de vida e tamanho (volume das operações) são variáveis comumente utilizadas nos estudos sobre a sobrevivência das empresas. A importância da idade da organização foi objeto dos estudos de Barron, West e Hannan, (1994), de Bruderl e Schussler (1990), de Fichman e Levinthal (1991) e de Levinthal (1991). A importância do tamanho é evidenciada nos trabalhos de Barnett e Amburgey (1990), de Hambrick e D'Aveni (1988) e de Wholey, Christianson e Sanchez (1992). As duas variáveis têm relação estreita com o processo de gestão estratégica induzido (ou por indução), expressando a busca contínua pelo aumento da eficiência no contexto de um ambiente concorrencial de relativa estabilidade.
Em uma análise mais rica, Bercovitz e Mitchell (2007) combinam proposições da teoria evolucionista da empresa (NELSON; WINTER, 1982), da ecologia das organizações (HANNAN; FREEMAN, 1989) e, em especial, da teoria da aprendizagem (COHEN; LEVINTHAL, 1990). Os autores consideram a variável tamanho mas enfatizam a dimensão escopo das atividades (diversificação), que está intimamente relacionada à trajetória de estratégia autônoma, conforme Burgelman e Grove (2007).

Empresas diversificadas (em produtos, em mercados, ou em ambos) possuem um conjunto mais amplo de rotinas organizacionais do que aquelas mais focalizadas (especializadas). Sob a perspectiva do conceito "capacidade de absorção" (COHEN; LEVINTHAL, 1990), a variedade de rotinas, de um lado, aumenta a capacidade de as organizações perceberem novas oportunidades e, de outro, aumenta as possibilidades de recombinação das rotinas existentes, visando à implementação de mudanças.

Quanto à sobrevivência das empresas, há ainda que considerar a possível influência da variável local (ou economia da localização).

\subsection{Idade e tamanho da empresa como bases da busca pela eficiência inerente ao processo de gestão estratégica "por indução"}

$\mathrm{Na}$ análise do efeito da aprendizagem pela experiência sobre o aumento da eficiência, Le Mens, Hannan e Pólos (2011) observam que o aprimoramento das competências (gerenciais e técnicas) está atrelado ao crescente engajamento da organização ao seu ambiente relevante, o que reforça a "familiaridade com o ambiente" que caracteriza o processo de gestão estratégica "por indução" proposto por Burgelman e Grove (2007).

Le Mens, Hannan e Pólos (2011) ressaltam que a noção de engajamento (HANNAN; PÓLOS; CARROLL, 2007) tem relação com o atendimento eficiente e eficaz da demanda, o qual está atrelado a: aprendizagem das idiossincrasias dos clientes; desenho/redesenho das características dos produtos/ serviços; e estabelecimento de uma identidade.

Carroll e Hannan (2000), tendo como referência Stinchcombe (1965), argumentam que um período de tempo considerável é necessário para uma empresa se estabelecer no mercado, envolvendo o desenvolvimento de conhecimento específico de rotinas organizacionais e a geração de confiança entre os membros internos à organização e entre ela e seus clientes e fornecedores. Durante esse período de desenvolvimento e de consolidação de competências, as organizações mais jovens estarão em desvantagem com relação às mais maduras e sujeitas a desafios que podem ser mais bem 
enfrentados pelas organizações já estabelecidas. À medida que a idade avança, a organização acumula mais experiência, seus membros aprendem a operar juntos e a compreender o ambiente competitivo.

A partir dos resultados de diversos estudos, Carroll e Hannan (2000) observam que empresas jovens tendem a apresentar maior taxa de mortalidade, sendo essa declinante com o aumento do tempo de vida. No entanto, alguns autores, como Baum (1989) e Hannan (1998), encontraram evidências de que, a partir de certa idade, a probabilidade de mortalidade da empresa volta a aumentar. Isso seria decorrência de uma espécie de "senilidade" à qual empresas antigas estariam sujeitas. Empresas antigas correriam o risco de ficarem atadas à inércia organizacional, incapazes de se adaptar a mudanças no ambiente competitivo. De um lado, a idade (tempo de vida) implica aprendizagem e estruturação no ambiente relevante. De outro, pode aumentar a inércia estrutural, tornando a empresa rígida e "obsoleta".

Sobre a relação entre idade e risco de mortalidade da empresa, vale a advertência de Van Wissen (2002), no sentido de que o efeito da idade da organização sobre a sobrevivência precisa ainda ser mais bem estudado.

Com base nessa argumentação, pode-se formular a seguinte hipótese:

Hipótese 1: O tempo de vida em t (1975, no caso deste estudo) não é um fator determinante quanto à probabilidade de uma pequena empresa sobreviver no tempo $(\mathrm{t}+\mathrm{n})$, no caso deste estudo, 2007.

Vale a pena destacar, como faz Van Wissen (2002), a dificuldade de separação do efeito das variáveis, tempo de vida e tamanho, dado que estão relacionadas. Recorrendo ao argumento de Steindl (1945), embora empresas possam nascer já grandes, outras morrem nos estágios iniciais de vida, caso em que, reconhecidamente, se inclui boa parte das pequenas empresas. Consequentemente, pode-se esperar que empresas antigas sejam também as de maior tamanho. Muitos dos efeitos que podem ser atribuídos à idade podem também ser atribuídos ao tamanho, e vice-versa. Porém a interação tempo de vida e tamanho na determinação da sobrevivência é complexa. Carroll e Hannan (2000) citam vários estudos que mostram resultados contraditórios, a partir da manutenção sob controle de uma das variáveis.

Uma das vantagens decorrentes do tamanho é a eficiência em custos alcançada por meio de mecanismos como: utilização de equipamentos especializados; economias de capital e retorno mais rápido, a partir da expansão operacional; aprofundamento da especialização dos empregados, a partir da intensificação da divisão do trabalho; e redução dos custos de overhead (administração e vendas).
Dobrev e Carroll (2003) acrescentam que os aumentos do tamanho e da participação no mercado são associados e levam à elevação do poder de negociação com clientes e fornecedores, além de facilitarem o acesso ao crédito e a fontes de capital. É ainda preciso considerar as barreiras à entrada em indústrias nas quais a razão entre escala mínima eficiente e tamanho de mercado é elevada. Essas condições representam vantagens competitivas e, consequentemente, contribuem para a continuidade da empresa no mercado.

Com base nessa argumentação, pode-se formular a seguinte hipótese:

Hipótese 2: A probabilidade de uma pequena empresa sobreviver no tempo $(\mathrm{t}+\mathrm{n}), 2007$, aumenta de acordo com o seu tamanho em t (1975).

Como a gestão estratégica "por indução" é "específica ao contexto atual", ficam evidentes as dificuldades (impedimentos) de transposições de processos e mecanismos decisórios para novos ambientes e mesmo de adaptação em situações nas quais o ambiente atual se altera profundamente. Se a concorrência se torna mais intensa e o ambiente é de mudanças significativas, o aprendizado com base na experiência (no tempo) não necessariamente se traduz em desempenho superior. Nesse sentido, é preciso aprofundar a discussão do processo de gestão estratégica autônomo, como proposto por Burgelman e Grove (2007).

\subsection{Escopo das atividades: amplitude do mercado e da linha de produtos (diversificação) como base do processo de gestão estratégica autônomo}

A estratégia de indução não é suficiente para a sobrevivência e expansão da empresa. Principalmente quando são requeridas capacidade de adaptação e capacitações para identificar e, principalmente, aproveitar novas oportunidades em condições de mudança no ambiente concorrencial.

Sobre as possibilidades e consequências da mudança organizacional, Haveman (1993) identifica duas vertentes: adaptação e seleção. Na primeira, constituída a partir de diferentes abordagens, a proposição central é que as organizações frequentemente se transformam e que a mudança está atrelada à adaptação da empresa ao ambiente.

Na segunda vertente, a proposta é que a mudança organizacional requer alterações mais profundas, com a destruição de práticas e estruturas existentes, isto é, implica uma ruptura, podendo representar uma ameaça à sobrevivência da organização. $\mathrm{O}$ autor sistematiza essa visão em torno do conceito "inércia estrutural", articulado a partir de duas proposições: baixa capacidade de mudança, associada a restrições de natureza interna e externa; e a perda de competência 
que pode resultar da transformação estrutural ou da reposição de pessoal.

Para Delacroix e Swamimatahan (1991), as duas vertentes se diferenciam de maneira acentuada quanto ao caráter otimista ou pessimista no que tange à mudança organizacional. Na primeira vertente, o otimismo é elevado, seja no que tange à habilidade que as empresas têm para promover mudanças, seja quanto à ideia de que a mudança organizacional é por si só benéfica. Em contraste, na segunda vertente, o pessimismo é elevado, tanto no que se refere às dificuldades da mudança quanto no que diz respeito aos perigos que a mudança representa, incluindo o possível aumento do risco de mortalidade da empresa.

Fica clara a conveniência de avançar a discussão na direção de abordagens intermediárias ou "conciliatórias", que explicitem as possibilidades e os limites da mudança organizacional. Insere-se nesse esforço a proposição de Jones $(2004,2005)$. Para o autor, na análise do processo de mudança (transformação) organizacional, dois pontos são essenciais: a percepção das pressões externas, associada à interação da empresa com o ambiente, por meio das suas atividades, produtos/serviços e da sua identidade com o ambiente, e a conciliação com uma "base de referência", que limita o grau de mudança, a partir da avaliação dos possíveis resultados.

O primeiro ponto significa o afastamento da concepção dos gestores como "agentes racionais". É o componente cognitivo da decisão e da ação, discutido amplamente em estudos sobre a aprendizagem organizacional, que ressalta a importância da experiência e do conhecimento acumulados no aguçamento da percepção de oportunidades e ameaças. Ademais, o processo de decisão envolve incursões de "tentativa e erro", de modo que os resultados não são previsíveis. O segundo ponto enfatiza as barreiras e a capacidade para a implementação de mudanças.

Para Jones (2005), os dois pontos são sintetizados no conceito capacidade de absorção, entendido por Cohen e Levinthal (1990) como a habilidade não só para reconhecer o valor da nova informação mas, também, absorvê-la e aplicá-la comercialmente (visando lucro). Ainda com relação à capacidade de absorção, Zahra e George (2002) definem a capacidade de absorção considerando dois componentes: a capacidade potencial de absorção e a capacidade potencial de realização. O potencial de absorção diz respeito à obtenção e assimilação de novos conhecimentos, ou seja, à capacidade (dos gestores) de perceber, obter, compreender e internalizar informações relevantes. O potencial de realização está associado às possibilidades abertas pelas rotinas operacionais (NELSON; WINTER, 1982) existentes para a combinação do conhecimento atual e de novos conhecimentos, de modo a gerar novos insights e a desenvolver novas competências.
Essas proposições abrem espaço para a discussão das diferenças existentes entre as empresas no tocante à capacidade de perceber oportunidades e de implementar mudanças que influenciam a longevidade.

Sob a perspectiva da percepção de oportunidades, Miller e Chen (1994) enfatizam a importância da diversidade de clientes e concorrentes. Organizações que disputam espaço com diferentes concorrentes e que buscam ser as selecionadas em um mercado com grande variedade de clientes tendem a aprender mais sobre as demandas e possibilidades do seu ambiente. Têm acesso a muitos tipos de informação, de múltiplas fontes, e estão expostas a ampla gama de opções competitivas. Em contraposição, organizações que competem em mercados estreitos, com concorrentes semelhantes, ou aquelas que têm somente um tipo de consumidor (cliente) atuam em um ambiente de aprendizagem mais estéril e homogêneo.

Nesse sentido, propõe-se a seguinte hipótese:

Hipótese 3: A probabilidade de uma pequena empresa sobreviver no tempo $(t+n), 2007$, aumenta de acordo com a amplitude geográfica do mercado em t (1975).

No entanto, como ressalta Jones (2004), o potencial de transformação da empresa depende da relação de complementaridade entre a capacidade potencial de absorção e a capacidade potencial de realização. Não basta perceber oportunidades. Para a mudança, para a evolução da empresa, são fundamentais as capacitações que permitem ajustar suas rotinas operacionais. A estabilidade (baixo grau de liberdade das rotinas operacionais) pode restringir a exploração de novas opções. A empresa é incapaz de responder apropriadamente aos estímulos emanados do ambiente, dada a rigidez das suas rotinas operacionais, o que pode afetar, como apontado entre outros por Langlois (1997), a permanência da empresa no mercado.

Com o mesmo sentido, Mukherjee, Mitchell e Talbot (2000) argumentam que uma linha de produção muito focada, definida em termos da limitação das possibilidades de produção, considerando um nível restrito de "requisitos de produção", limita a capacidade de absorção para a adaptação quanto a requisitos não relacionados às tarefas já praticadas. Entendendo a linha de produção como uma "unidade organizacional", visualizada como uma coleção de rotinas (NELSON; WINTER, 1982), fica claro que uma empresa com produção focada desenvolve um conjunto de rotinas de produção que envolve um conjunto limitado de habilidades (processamentos, fluxo de informações e fluxo de materiais). Embora suficientes nas condições correntes, quando houver necessidade de se mudarem as atividades, diante de mudanças no ambiente concorrencial, o conjunto limitado de rotinas (a natureza das rotinas operacionais) pode restringir a capacidade de mudança e de evolução das unidades organizacionais. 
Conforme proposto por Cohen e Levinthal (1990), é necessário reconhecer os novos requisitos, em termos das rotinas que serão necessárias; avaliar o repertório atual de rotinas para identificar potenciais elementos de recombinação; identificar recursos externos que serão requeridos pelas novas rotinas, e utilizar elementos apropriados das rotinas existentes em conjunto com os recursos externos. Em síntese, a linha de produção tende a desenvolver uma capacidade de absorção fortemente correlacionada ao conjunto corrente (atual) de habilidade (rotinas operacionais).

Com base nessa argumentação, pode-se propor a seguinte hipótese:

Hipótese 4: A probabilidade de uma pequena empresa sobreviver no tempo $(\mathrm{t}+\mathrm{n}), 2007$, aumenta de acordo com a amplitude da linha de produtos em t (1975).

\subsection{Algumas considerações sobre os efeitos das economias de localização na sobrevivência das empresas}

A presença de aglomerações industriais (dimensão geográfica ou regional) é outra fonte de explicação para heterogeneidade quanto ao desempenho das organizações. Os benefícios decorrentes da concentração de uma indústria ou grupo de indústrias relacionadas em uma região emergem, conforme destacado por Wennberg e Lindqvist (2010), de três fontes: custos de transporte (WEBER, 1957), economias externas (MARSHALL, 1982); e efeitos sociocognitivos (SORENSEN; SORENSON, 2003).

Em levantamento dos estudos focados na influência das economias de localização sobre a sobrevivência e desempenho das empresas, realizado por Fritsch, Noseleit e Schindele (2010) e por Wennberg e Lindqvist (2010), os resultados empíricos não foram conclusivos. Alguns estudos indicaram efeitos positivos do "estar localizado em uma aglomeração de empresas" sobre a sobrevivência. Fritsch, Noseleit e Schindele (2010) citam a pesquisa efetuada por Fotopoulos e Louri (2000), na Grécia. Wennberg e Lindqvist (2010) citam a pesquisa de Beaudry e Swann (2001), na Grã-Bretanha. Entretanto, os resultados de outros estudos, Audretsch e Vivarelli (1995) e Gerlach e Wagner (1994), citados por Fritsch, Noseleit e Schindele (2010), mostram significativos efeitos negativos. Wennberg e Lindqvist (2010) citam o estudo de Globerman, Shapiro e Vining (2005) sobre o crescimento das vendas e a sobrevivência de empresas de tecnologia de informação no Canadá, que constatou efeitos positivos moderados da localização sobre o crescimento das vendas e sobre a sobrevivência das empresas.

Uma possível explicação para a localização em aglomerados setoriais de empresas não ser um fator com efeitos positivos sobre a sobrevivência das empresas é apontada por Wennberg e Lindqvist (2010), com base nos estudos de Beaudry e Swann (2001), Folta, Cooper e Baik (2006) e Prevezer (1997). Os efeitos benéficos sobre a eficiência e a inovação podem ser superados pelos efeitos adversos derivados do número elevado de empresas, que leva a uma intensa concorrência por recursos. Além disso, deve-se considerar que o número elevado de empresas concentradas em uma localidade, com produtos pouco diferenciados, conduz a uma concorrência acirrada, com efeitos negativos sobre a rentabilidade das empresas e do setor no local.

Com base nessa argumentação, pode-se propor a seguinte hipótese:

Hipótese 5: A probabilidade de uma pequena empresa sobreviver no tempo $(t+n), 2007$, não é influenciada de forma significativa pelo fato de estar localizada em aglomeração de empresas de um mesmo setor de atividade em t (1975).

\section{Metodologia}

Trata-se de uma pesquisa descritiva, que tem como foco testar o conjunto de hipóteses colocadas ao longo das seções 3.1, 3.2 e 3.3. Segundo Cervo, Bervian e Silva (2007), as pesquisas descritivas têm o objetivo de propiciar maior entendimento sobre a frequência com que um fenômeno ocorre, sua correlação e conexão com outros fenômenos.

Para atingir esse objetivo, a estratégia de pesquisa, seguindo o conceito de "concepção de pesquisa" de Ragin (1994, p. 191), consiste em: "[...] um plano para a coleta e a análise de evidências que irá possibilitar ao investigador responder quaisquer questões que ele tenha colocado". A investigação neste trabalho está em consonância com essa concepção.

\subsection{Base de dados}

Para este estudo foi construído um banco de dados com informações do universo das pequenas empresas industriais da região de Campinas, em 1975 e em 2007, compreendendo 23 municípios. São consideradas pequenas empresas aquelas com até 100 empregados, de acordo com a classificação do SEBRAE. As informações referentes a 1975 foram obtidas por meio do Cadastro Industrial da Região de Campinas realizado pelo Centro Técnico Econômico de Assessoria Empresarial da Universidade Estadual de Campinas (CTAE/Unicamp). Os dados e informações referentes a 2007 foram extraídos do Cadastro da Secretaria da Fazenda do Estado de São Paulo (SEFAZ).

O Cadastro do CTAE/Unicamp contém informações sobre: ramos de atividade, porte, linha de produtos e abrangência de mercado. As informações disponíveis permitiram caracterizar as empresas quanto aos quatro 
fatores mencionados (idade, tamanho, escopo das atividades e localização).

Os dados da Secretaria da Fazenda do Estado de São Paulo (SEFAZ), levantados em 2007, complementam o banco de dados. Esses dados permitiram identificar se as empresas constantes no Cadastro Industrial da Região de Campinas, em 1975, continuavam ativas em 2007.

\subsection{Técnica estatística: regressão logística}

A análise ao longo deste estudo requereu a separação das pequenas empresas industriais em duas classes, as sobreviventes e as não sobreviventes, e a identificação das variáveis que potencialmente explicam essa dicotomia. Entre as técnicas estatísticas existentes, foram consideradas mais adequadas a Análise Discriminante e a Regressão Logística, ambas na classe de métodos estatísticos multivariados (HAIR et al., 2007; SHARMA, 1996; PREARO, 2008).

Optou-se pela análise da regressão logística tendo em vista que a natureza do fenômeno tem aderência à forma matemática da função logística, ou seja, com pontos de saturação inferiores e superiores. Mais precisamente, considerando a variável tempo de vida, essa técnica permite identificar a tendência quanto à sobrevivência tanto entre pequenas empresas com pouca idade quanto entre pequenas empresas com muitos anos de vida. No longo prazo, a tendência é de que ambas não sobrevivam. Ademais, segundo Garson (2007), a Regressão Logística é mais utilizada, pois, ao contrário da Análise Discriminante, não assume a linearidade das relações, não assume que o erro seja normalmente distribuído e não faz exigência quanto à normalidade da distribuição multivariada, além de relaxar a premissa de homoscedasticidade.

\subsubsection{Avaliação dos dados quanto às premissas da análise de regressão logística}

Para a análise estatística dos dados e dos resultados foi utilizado o software aplicativo Statistical Package for the Social Sciences (SPSS). Inicialmente, visando verificar se os dados atendiam às premissas da análise de regressão logística, foram considerados os seguintes aspectos: sensibilidade ao tamanho da amostra, multicolinearidade, presença de observações atípicas e normalidade.

Segundo Peduzzi et al. (1996), o tamanho da amostra adequado para a aplicação da regressão logística é de 10 observações para cada variável integrante do modelo. Na presente pesquisa, o número de observações para cada variável foi de 499,66. O número de observações foi de 2.998 (correspondente ao número de pequenas empresas constantes do Cadastro Industrial da Região de Campinas em
1975). O modelo em análise possui cinco variáveis independentes e uma variável dependente.

A ausência de multicolinearidade foi verificada pela estatística VIF (Fator de Inflação da Variância), sugerida por Hair et al. (2007) e Garson (2007), situando-se no intervalo 1,064 a 1,317. Segundo Gujarati (2000), um VIF maior do que cinco indica a presença de multicolinearidade grave.

Segundo Prearo (2008), observações atípicas ou extremas (outliers) são aquelas substancialmente diferentes das outras, que provocam alterações no resultado da análise e causam violações de normalidade. No que se refere aos outliers, foi adotado como critério o "corte em três desvios no score padronizado" das variáveis idade e tamanho. Como resultado desse procedimento, optou-se por retirar da amostra pequenas empresas com idade superior a 35 anos e com um número total de empregados superior a 63, de forma a concentrar a análise no extrato mais representativo das pequenas empresas. Com isso, o número total de observações considerado na análise passou de 2.998 para 2.811 .

As variáveis originais foram padronizadas e foi feito o teste Kolmogorov-Smirmov para verificação da normalidade. Nenhuma das variáveis apresentou aderência à distribuição normal. Dessa forma, estabeleceu-se que o modelo Logit era o mais adequado para a análise dos dados.

\subsubsection{Modelo Logit e especificação das variáveis}

A taxa de sobrevivência das empresas foi analisada segundo o ano de início das atividades, tendo como referência os dois bancos de dados. Foi utilizado um modelo Logit - Equação 1 -, com o objetivo de identificar as variáveis que contribuíram para a probabilidade de uma empresa estar em atividade em 2007.

$$
L_{i}=\ln \left(\frac{P_{i}}{1-P_{i}}\right)=\mathrm{x} \beta+u_{i}
$$

onde $P /\left(1-P_{i}\right)$ representa a razão entre a probabilidade de uma empresa $i$ estar em atividade em 2007 e a probabilidade de não estar em operação nesse mesmo ano. Além disso, $\mathbf{x}$ consiste no vetor de variáveis explicativas do modelo; $\beta$ expressa o vetor de parâmetros que refletem o impacto de variações de $\mathbf{x}$ na probabilidade de continuidade da empresa; e $u_{i}$, o termo de erro que segue distribuição normal com média zero e variância constante.

Os parâmetros da regressão foram estimados pelo método da máxima verossimilhança, dado que $P_{i}$ é não linear em $\mathbf{x}$ e em $\beta$. Vale notar que os parâmetros $\beta$ indicam efeito de mudanças nas variáveis explicativas $\left(X_{i}\right)$, presentes em $\mathbf{x}$, sobre o logaritmo natural da razão de probabilidades $\left(L_{i}\right)$. Assim, para 
mensurar o impacto de variações $X_{i}$ sobre $P_{i}$ é preciso transformar os valores estimados para $\beta$ tomando-se o antilogaritmo do Logit estimado na Equação 1. Ou seja (Equação 2):

$$
P_{i}=E(Y=1 \mid \mathrm{x})=1-E(Y=0 \mid \mathrm{x})=\frac{e^{\mathrm{x}^{\prime} \beta}}{1+e^{\mathrm{x}^{\prime} \beta}}
$$

onde $Y$ é a variável dependente binária, a qual assume valor igual a 1 se a empresa $i$ está em operação em 2007 e valor 0 (zero) caso contrário.

As variáveis explicativas, $X_{i}$, presentes no vetor $\mathbf{x}$ foram: a) idade da empresa $i$ em 1975; b) tamanho (volume das operações), utilizando como proxy o número de funcionários da empresa $i$ em 1975; c) amplitude da linha de produtos representada por uma variável dummy, que assume valor igual a 1 se a empresa $i$ fabricava mais de uma linha de produtos e valor 0 (zero) caso contrário; d) abrangência em termos de mercado, sendo isso captado por um índice de diversificação dos mercados geográficos atendidos pela empresa em 1975 - assume valor 1 se as vendas eram estritamente locais, 2 se existiam vendas regionais, 3 se eram verificadas vendas para outros estados e 4 se eram observadas vendas para mercados externos; e) economia de localização, sendo avaliada pelo fato de a empresa pertencer ou não a uma região e mercado que se caracterizavam como uma aglomeração setorial de empresas. Tal variável também foi expressa por uma dummy - com valor igual a 1 se o município possuía tal característica e 0 (zero) caso contrário.

\section{Apresentação e análise dos resultados}

Foram incluídos no modelo, para analisar a sobrevivência a longo prazo, 2.811 pequenas empresas industriais, que representam $93,76 \%$ dos casos. Foram excluídos 6,24\% dos casos tendo em vista o seu caráter atípico, conforme já explicado. A partir de tais dados, constantes na Tabela 1, observa-se que a taxa de sobrevivência das pequenas empresas industriais no período compreendido entre 1975 e 2007 foi relativamente baixa, em torno de $23 \%$. Entretanto, o fato de terem permanecido no mercado, passando pelas várias crises que o país enfrentou entre as décadas de 1970 e o início da década de 2000, revela capacidade de adaptação, resistência e competitividade das empresas sobreviventes.

Para verificação da qualidade do modelo logístico foram aplicadas as estatísticas -2 Log likelihood (verossimilhança logarítmica), Cox \& Snell $\mathrm{R}^{2} \mathrm{e}$ Nagelkerke $\mathrm{R}^{2}$, apresentadas na Tabela 2. Essas estatísticas expressam a capacidade de explicação da variável dependente pelas variáveis independentes selecionadas. Os valores das estatísticas de $R^{2}$ são relativamente baixos, sinalizando que há outras variáveis que influenciam a sobrevivência das pequenas empresas, não consideradas neste estudo. Isso significa que não é possível utilizar o modelo como um instrumento de predição. Esse fato não invalida os resultados obtidos, tendo em vista que o objetivo do estudo é identificar a influência de cada uma das variáveis independentes sobre a variável dependente e não o quanto cada uma delas influencia. A aplicação do modelo permitiu constatar interação positiva das variáveis de forma consistente com diversas proposições encontradas na literatura sobre o tema.

Ainda quanto à avaliação da qualidade do ajuste do modelo logístico, foi aplicada a estatística de Hosmer e Lemeshow, que mede a correspondência entre os valores reais e os previstos da variável dependente, utilizada para testar as classificações esperadas e observadas. A hipótese nula para esse teste é de que as classificações previstas são iguais às observadas. A expectativa é aceitar (não rejeitar) a hipótese nula. A estatística qui-quadrado para o referido teste é de 4,148, com nível de significância de 0,844 e 8 graus de liberdade (df). Esse resultado pertence à área de não rejeição da hipótese nula.

A Tabela 3 apresenta a "matriz de classificação do modelo", a qual aponta para uma medida do nível de acerto do modelo, isto é, indica quantitativamente o quanto o modelo "acertou" na estimativa da variável dependente. Segundo Hair et al. (2007), para pesquisas exploratórias são aceitáveis níveis de acerto acima de $60 \%$.

Tabela 1. Taxa de sobrevivência das empresas industriais da região de Campinas 1975-2007.

\begin{tabular}{ccc}
\hline Ativa em 2007 & $\begin{array}{c}\text { Quantidade de } \\
\text { empresas }\end{array}$ & $\%$ \\
\hline Sim & 648 & 23,05 \\
Não & 2.163 & 76,95 \\
Total & 2.811 & 100,00 \\
\hline
\end{tabular}

Fonte: elaborada pelos autores.

Tabela 2. Indicadores de ajuste do modelo logístico.

\begin{tabular}{cccc}
\hline Passo & $\begin{array}{c}\text { Verosimilhança } \\
\text { logarítmica }\end{array}$ & $\begin{array}{c}\text { Cox } \& \\
\text { Snell } \mathbf{R}^{\mathbf{2}}\end{array}$ & $\begin{array}{c}\text { Nagelkerke } \\
\mathbf{R}^{\mathbf{2}}\end{array}$ \\
\hline 1 & $2.784,307$ & 0,051 & 0,078 \\
\hline
\end{tabular}

Fonte: elaborada pelos autores.

Tabela 3. Matriz de classificação do modelo observado.

\begin{tabular}{lccc}
\hline Observado & \multicolumn{3}{c}{ Previsão } \\
\cline { 2 - 4 } & $\begin{array}{c}\text { Inativas } \\
(\mathbf{2 0 0 7 )}\end{array}$ & $\begin{array}{c}\text { Ativas } \\
\mathbf{( 2 0 0 7 )}\end{array}$ & \% acerto \\
\hline Inativas & 2.163 & 42 & 98,1 \\
Ativas & 570 & 36 & 5,9 \\
\% total & & & 78,9 \\
\hline
\end{tabular}

Fonte: elaborada pelos autores. 
A avaliação do poder discriminatório da matriz de classificação, quando comparada com um modelo de acertos ao acaso, pode ser feita a partir da estatística $Q$ de Press, que resulta em uma estatística qui-quadrado dada por (Equação 3):

$$
\mathrm{Q} \text { de Press }=(N-n K)^{2} / N(K-1)
$$

onde $N$ representa o número de casos estudados, $n$ o número de acertos do modelo e $K$ é o número de grupos estudados. $O$ valor da estatística qui-quadrado para um nível de significância de 1\% com 1 grau de liberdade é 6,63. Considerando-se que o valor calculado é de 895,96, maior do que o valor tabelado, conclui-se que as previsões geradas no modelo logístico estimado são significativamente melhores do que as estimativas obtidas ao acaso.

$\mathrm{Na}$ Tabela 4 apresentam-se as estimativas dos coeficientes Beta $(\beta)$ - coeficientes estimados para as variáveis independentes - e os testes de significância. Esses coeficientes podem ser avaliados em termos de significância estatística com base na estatística de Wald (HAIR et al., 2007), definida como o quadrado da razão entre o coeficiente logístico estimado e o seu erro padrão. Os resultados apontam que os coeficientes de todas as variáveis incluídas no modelo são estatisticamente diferentes de zero.

Quanto às variáveis tamanho, amplitude da linha de produtos e abrangência em termos de mercados, foram confirmadas a hipótese 2 , proposta na seção 3.1, e as hipóteses 3 e 4, propostas na seção 3.2. Tais variáveis são consideradas discriminantes para a sobrevivência a longo prazo das pequenas empresas industriais, uma vez que a significância é inferior a $5 \%$. Quanto à variável tempo de vida da empresa, verificou-se que não é discriminante, conforme aventado na hipótese 1, proposta na seção 3.1. Da mesma forma, a variável economia de localização não é discriminante, o que é pertinente com a proposição da hipótese 5, apresentada na seção 3.3.

Observando-se o valor de $\beta$ (coeficiente da regressão logística) expresso na Tabela 4, é possível ainda realizar uma classificação das variáveis discriminantes. Nota-se que a amplitude da linha de produtos e a abrangência em termos de mercado são as variáveis mais importantes, seguidas pela variável tamanho.

É significativo o fato de os resultados da pesquisa indicarem que a variável tamanho tem relação positiva com a sobrevivência das empresas consideradas. No entanto, os fatores mais importantes para a elevação da probabilidade de sobrevivência a longo prazo desse porte de empresa foram a amplitude da linha de produtos (diversificação de produtos) e a abrangência em termos de mercado.

A relevância da diversificação de produtos e de mercados na longevidade das pequenas empresas industriais, como de qualquer outra empresa, está ligada, conforme destacado na literatura examinada, à permanência no mercado e aos resultados obtidos. Tais resultados expressam a evolução temporal do desempenho organizacional, que depende de fatores internos à empresa, sobre os quais há mais poder de gestão, e externos, sobre os quais os dirigentes têm pouco ou nenhum poder de gestão.

A definição de estratégias é um dos meios pelos quais as empresas buscam conquistar e manter posições no mercado. Como destacado na literatura, em condições de estabilidade do ambiente concorrencial, estratégias indutivas são adequadas. Entretanto, em situação de mudanças significativas nesse ambiente é necessário avançar na direção de estratégias autônomas. Esse processo é bastante dificultado em pequenas empresas, dados os limitados recursos (monetários e de pessoal).

As características das PEs, em especial aquelas relativas às restrições quanto ao acesso a fontes de conhecimento e de financiamento, representam forte desvantagem relativamente às organizações com maior disponibilidade de capital. Isso porque, identificada uma oportunidade, a passagem dessa etapa à concretização em produtos e em receita demanda investimentos, nem sempre disponíveis para as empresas menores. Portanto, ao explicarem-se a sobrevivência e o crescimento das pequenas empresas há que incluir não só a capacidade de identificar oportunidades mas também as condições que permitem sua transformação em negócios efetivos.

Tabela 4. Resultados da estimação do modelo logit.

\begin{tabular}{|c|c|c|c|c|c|c|}
\hline Variável & $\beta$ & SE & WALD & df & Sig. & $\begin{array}{l}\operatorname{Exp}(B) \\
\text { (Poder) }\end{array}$ \\
\hline 1. Idade & .010 & .006 & 2.447 & 1 & .118 & 1.010 \\
\hline 2. Tamanho & .029 & .004 & 49.849 & 1 & .000 & 1.029 \\
\hline 3. Amplitude da linha de produtos & .265 & .104 & 6.454 & 1 & .011 & 1.303 \\
\hline 4. Abrangência em termos de mercados & .265 & .060 & 19.745 & 1 & .000 & 1.303 \\
\hline 5. Economia de localização & -.057 & .125 & .208 & 1 & .648 & .945 \\
\hline Constante & -2.215 & .132 & 280.203 & 1 & .000 & .109 \\
\hline
\end{tabular}

Fonte: elaborada pelos autores. 
Levando-se isso em consideração, na análise dos resultados da pesquisa deve ser enfatizada, como fator relevante para a longevidade das pequenas empresas, a capacidade de perceber oportunidades e aproveitá-las dos dirigentes, utilizando os recursos disponíveis. Esse é um aspecto explorado em algumas abordagens sobre o crescimento e a sobrevivência de empresas, as quais destacam a importância do perfil dos dirigentes no conjunto de fatores internos que podem ser utilizados para explicar o desempenho das empresas. Nesses estudos é realçada também a capacidade de construir uma identidade própria, a qual distingue a empresa das demais, isto é, também nesse caso empresas que disputam espaço no mesmo setor não concorrem em iguais condições competitivas.

Pela natureza eminentemente qualitativa do mencionado fator, não há como incluí-lo em análise estatística quantitativa. No entanto, não se pode desconsiderar que, diante das mesmas condições externas, as particulares competências de cada empresa levam a ações estratégicas diferentes e com efeitos sobre a sobrevivência. Empresas com equipes dirigentes dotadas de aptidões, ideias originais e flexibilidade apresentam como característica central a ampliação do horizonte de crescimento. Tendo mais chances de escolha, não serão atraídas para segmentos nos quais a taxa de lucro é comprimida pela presença de grande número de empresas com poucas possibilidades de diferenciação e, por consequência, são beneficiadas pelo aumento da probabilidade de sobrevivência. Hipótese confirmada na análise estatística.

Com relação à variável tamanho, embora discriminante, não se apresentou com o mesmo destaque da diversificação. No entanto, pode ser considerado um dos fatores que aumentam a probabilidade de sobrevivência das pequenas empresas.

Nas PEs, pelas suas características, a geração de lucro está sujeita a restrições mais severas do que nas grandes, tanto no que se refere à exposição e à capacidade de reação às imposições do ambiente externo, quanto pelas dificuldades associadas aos fatores internos. Além disso, como as PEs têm menor poder de negociação com fornecedores, estão mais sujeitas às pressões de custo. Nas relações com clientes, estão mais sujeitas a pressões sobre os preços. Em termos financeiros, o tamanho tem relação direta com a capacidade de obtenção de receita total para cobrir os custos e propiciar margem de lucro suficiente para o crescimento. Além disso, o tamanho confere outras vantagens ligadas à sobrevivência, por meio do aprimoramento das "rotinas organizacionais" e da ampliação da rede de relações com fornecedores e clientes, conforme salientado na literatura.

Conforme também enfatizado na literatura por alguns autores, o aumento do tamanho tende a provocar alterações nas atividades gerenciais, que se tornam mais complexas, tais como planejamento, mensuração e mecanismos de controle voltados para o aumento da eficiência. Cabe destacar que, com o crescimento (aumento de tamanho), a empresa se desloca para um novo patamar de competição, no qual enfrentará novas exigências em termos do padrão de concorrência e de atualização tecnológica.

Em síntese, o maior tamanho está associado à acumulação de recursos (evolução temporal do desempenho organizacional, favorecida pela cumulatividade). Aumenta-se a resistência das pequenas empresas que conseguem crescer, reduzindo-se as assimetrias que as desfavorecem e acentuando-se as vantagens do tamanho, inclusive poder no mercado.

Com relação aos resultados dos testes estatísticos a respeito da relação idade-sobrevivência a longo prazo da empresa, não são surpreendentes pois, como já apontado na literatura, diversos estudos não são conclusivos a esse respeito. A expectativa, fundada em proposições, em parte da literatura, sobre o tema, levaria a que empresas mais antigas teriam maior probabilidade de sobreviver que empresas mais novas, dadas as condições mais favoráveis para inserção no mercado, melhores redes e acesso a recursos. No entanto, em outra parcela da literatura argumentos defendem que, com a passagem do tempo, as empresas tendem a se tornar mais "rígidas". Superar essa rigidez exige recursos e implica custos que as pequenas não têm como suportar. Considerando que o período ao qual se referem os dados e as informações para este estudo (1975-2007) foi marcado por profundas turbulências no ambiente concorrencial, é evidente a importância de mudanças por parte das empresas. As pequenas empresas mais antigas podem ter enfrentado os efeitos da "senilidade": a idade teria sido traduzida em inflexibilidades, que impediram a implementação das mudanças estratégicas necessárias, levando ao desaparecimento da empresa.

\section{Conclusões}

Considerando o objetivo principal da pesquisa, o estudo apresenta duas principais contribuições para a compreensão da longevidade de pequenas empresas.

Em primeiro lugar, evidenciou que a amplitude da linha de produtos, a abrangência em termos de mercado e o tamanho exercem influência sobre a probabilidade de sobrevivência das pequenas empresas industriais a longo prazo. Essa conclusão é importante, pois a literatura que examina a influência da diversificação sobre a sobrevivência das pequenas empresas ainda é muito reduzida. A análise evidenciou que a diversificação exerce um papel fundamental sobre o "capital organizacional", com efeitos benéficos em ambientes instáveis.

Em segundo lugar, os resultados do estudo indicam que o tamanho da empresa interfere na probabilidade 
de sobrevivência a longo prazo das PEs industriais. A influência do tamanho extrapola a esfera financeira, envolvendo aspectos organizacionais e a inserção em redes de relações com fornecedores e clientes, que compõem o denominado "capital organizacional", essência da sobrevivência. Como a pesquisa não considerou a interface entre a diversificação e o tamanho, é necessário examiná-la em estudos posteriores, em particular investigar se as vantagens da diversificação emergem independentemente das vantagens associadas ao tamanho ou, ao contrário, se a empresa deve adquirir tamanho suficiente para poder usufruir dos benefícios da diversificação. Uma questão instigante poderia ser: em que medida a existência de uma acumulação mínima de recursos - financeiros e humanos - é uma condição para o processo de diversificação?

Ficou evidente a necessidade de empreender novos estudos, de caráter longitudinal, com o objetivo de explorarem-se as mudanças estratégicas empreendidas, as fases de transição e os caminhos seguidos. Para tanto, seria necessário acompanhar a trajetória das empresas e analisarem-se os estágios que percorreram em seu processo de desenvolvimento.

\section{Referências}

AUDRETSCH, D. et al. First Section of the Annual Report on EU Small and Medium-size Enterprises. EIM Business \& Policy Research, jan. 2009. Disponível em: <http://ec.europa.eu/enterprise/policies/sme/files/ craft/sme_perf_review/doc_08/spr08_annual_reporten. pdf $>$. Acesso em: 20 ago. 2010.

AUDRETSCH, D. B.; VIVARELLI, M. New-firm formation in Italy: a first report. Economics Letters, v. 48, n. 1, p. 77-81, 1995. http://dx.doi. org/10.1016/0165-1765(94)00587-R

BARNETT, W. P.; AMBURGEY, T. L. Do larger organizations generate stronger competition? In: SINGH, J. V. (Ed.). Organizational Evolution: New Directions. Newbury Park: Sage, 1990. p. 246-248.

BARRON, D. N.; WEST, E.; HANNAN, M. T. A time to grow and a time to die: growth and mortality of credit unions in New York City 1914-1990. American Journal of Sociology, v. 100, n. 2, p. 196-241, 1994. http:// dx.doi.org/10.1086/230541

BAUM, J. A. C. Liabilities of Newness, Adolescence, and Obsolescence: Exploring Age Dependence in the Dissolution of Organizational Relationships and Organizations. In: ANNUAL CONFERENCE OF THE ADMINISTRATIVE SCIENCE ASSOCIATION OF CANADÁ, 10., 1989. Ottawa. Proceedings... Montreal: Université du Québec, 1989. v. 10, p. 1-10.

BEAUDRY, C.; SWANN, P. Growth in industrial clusters: a bird's eye view of the United Kingdom. Stanford Institute for Economic Policy Research, 2001. Discussion Paper 00-38.

BERCOVITZ, J.; MITCHELL, W. When is More Better? The impact of business scale and scope on long-term business survival, while controlling for Profitability. Strategic
Management Journal, v. 28, n. 1, p. 61-79, 2007. http://dx.doi.org/10.1002/smj.568

BRUDERL, J.; SCHUSSLER, R. Organizational mortality: the liabilities of newness and adolescence. Administrative Science Quarterly, v. 35, n. 4, p. 530-547, 1990. http://dx.doi.org/10.2307/2393316

BURGELMAN, R. A. Intraorganizational ecology of strategy making and organizational adaptation: theory and field research. Organization Science, v. 2, n. 3, p. 239-262, 1991. http://dx.doi.org/10.1287/orsc.2.3.239

BURGELMAN, R. A.; GROVE, A. S. Let chaos reign, then rein in chaos - repeatedly: managing strategic dynamics for corporate longevity. Strategic Management Journal, v. 28, n. 10, p. 965-979, 2007. http://dx.doi.org/10.1002/ smj.625

CARROLL, G. R.; HANNAN, M. T. The demography of corporations and industries. Princeton: Princeton University Press, 2000.

CERVO, A. L.; BERVIAN, P. A.; SILVA, R. Metodologia científica. 6. ed. São Paulo: Pearson Prentice Hall, 2007.

COHEN, W.; LEVINTHAL, D. Absorptive capacity: a new perspective on learning and innovation. Administrative Science Quarterly, v. 35, n.1, p. 128-152, 1990. http:// dx.doi.org/10.2307/2393553

DELACROIX, J.; SWAMINATHAN, A. Cosmetic, speculative and adaptive organizational change in the wine industry. A longitudinal study. Administrative Science Quarterly, v. 36, n. 4, p. 631-661, 1991. http:// dx.doi.org/10.2307/2393277

DOBREV, S. D.; CARROLL, G. R. Size (and competition) among organizations: modeling scale-based selection among automobile producers in four major countries, 1885-1981. Strategic Management Journal, v. 24, n. 6, p. 541-558, 2003. http://dx.doi.org/10.1002/ smj.317

FICHMAN, M.; LEVINTHAL, D. A. Honeymoons and the liability of adolescence: a new perspective on duration dependence in social and organizational relationships. Academy of Management Review, v. 16 , n. 2 , p. 442-468, 1991.

FOLTA, T. B.; COOPER, A. C.; BAIK, Y. S. Geographic cluster size and firm performance. Journal of Business Venturing, v. 21, n. 2, p. 217-242, 2006. http://dx.doi. org/10.1016/j.jbusvent.2005.04.005

FOTOPOULOS, G.; LOURI, H. Location and survival of new entry. Small Business Economics, v. 14, n. 4 , p. 311-21, 2000. http://dx.doi. org/10.1023/A:1008180522759

FRITSCH, M.; NOSELEIT, F.; SCHINDELE, Y. Success or failure? A multi-dimensional analysis of business-, industry- and region-specific determinants of survival. 2010. Disponível em: <http://www.re.wu. ac.at/ersa/ersaconfs/ersa10/ERSA2010>. Acesso em: 20 abr. 2012

GARSON, G. D. Discriminant analysis, from statenote. Topics in multivariate analysis. 2007. Disponível em: $<$ http://www2.chass.nesu.edu/garson/pa765.statenote. html>. Acesso em: 20 jul. 2010.

GERLACH, K.; WAGNER, J. Regional differences in small firm entry in manufacturing industries: Lower Saxony 1979-1991. Entrepreneurship and Regional 
Development, v. 6, n. 1, p. 63-80, 1994. http://dx.doi. org/10.1080/08985629400000004

GLOBERMAN, S.; SHAPIRO, D.; VINING, A. Clusters and intercluster spillovers: their influence on the growth and survival of Canadian information technology firms. Industrial and Corporate Change, v. 14, n. 1, p. 27-60, 2005. http://dx.doi.org/10.1093/icc/dth043

GUJARATI, D. N. Econometria Básica. 3. ed. São Paulo: Makrom Books, 2000.

HAIR, J. F. et al. Análise multivariada de dados. 5. ed. Porto Alegre: Bookman, 2007.

HAMBRICK, D. C.; D'AVENI, R.A. Large corporate failures as downward spirals. Administrative Science Quarterly, v. 33, n. 1, p. 1-23, 1988. http://dx.doi. org/10.2307/2392853

HANNAN, M. Rethinking age dependence in organizational mortality: logical formalizations. American Journal of Sociology, v. 104, n. 1, p. 126-164, 1998. http://dx.doi. org/10.1086/210004

HANNAN, M. T.; FREEMAN, J. Organizational ecology. Cambridge: Harvard University Press, 1989.

HANNAN, M. T., PÓLOS, G. R.; CARROLL, G.R. Logics of organization theory: audiences, codes and ecologies. Princeton: Princeton University Press, 2007.

HAVEMAN, H. A. Organizational size and change: diversification in the saving and loan industry after deregulation. Administrative Science Quarterly, v. 38, n. 1, p. 20-50, 1993. http://dx.doi.org/10.2307/2393253

HEADD, B.; KIRCHHOFF, B. Small Business Growth: Searching for Stylized Facts. Small Business Administration and New Jersey Institute of Technology, Oct 2007. Working Paper.

JONES, C. An alternative view of small firm adaptation. Journal of Small Business and Enterprise Development, v. 11, n. 3, p. 362-370, 2004. http:// dx.doi.org/10.1108/14626000410551618

JONES, C. Firm Transformation: advancing a Darwinian perspective Management Decision, v. 43. n. 1, p. 13-25, 2005. http://dx.doi. org/10.1108/00251740510572452

LANGLOIS, R. N. Cognition and capabilities: opportunities seized and missed in the history of the computer industry, In: GARUD, R.; NAYYAR, P.; SHAPIRA, Z. (Eds.). Technological Entrepreneurship: oversights and foresights. New York: Cambridge University Press, 1997. p. 320-355. http://dx.doi.org/10.1017/ CBO9780511896613.007

LE MENS, G.; HANNAN, M. T.; PÓLOS, L. Founding Conditions, Learning, and Organizational Life Chances: Age Dependence Revisited. Administrative Science Quarterly, v. 56, n. 1, p. 95-126, 2011. http://dx.doi. org/10.2189/asqu.2011.56.1.095

LEVINTHAL, D. Random walks and organization mortality. Administrative Science Quarterly, v. 36, n. 3, p 397-420, 1991. http://dx.doi.org/10.2307/2393202

MARSHALL, A. Princípios de economia tratado introdutório. São Paulo: Abril Cultural, 1982.

MILLER, D.; CHEN, M.-J. Sources and consequences of competitive inertia: a study of competitive inertia: a study of US airline industry. Administrative Science
Quarterly, v. 39, n. 1, p. 1-23, 1994. http://dx.doi. org/10.2307/2393492

MUKHERJEE, A.; MITCHELL, W.; TALBOT, F. B. The impact of new manufacturing requirements on production line productivity and quality at a focused factory. Journal of Operations Management, v. 18, n. 2, p. 139-168, 2000. http://dx.doi.org/10.1016/ S0272-6963(99)00023-6

NAJBERG, S.; PUGA, F. P.; OLIVEIRA, P. A. S. Análise da sobrevivência das firmas brasileiras. Informe-se (BNDES), n. 46, p. 1-8, ago. 2002.

NELSON, R.; WINTER, S. An evolutionary theory of economic change. Cambridge: Harvard University Press, 1982.

PEDUZZI, P. J. et al. A simulation study of the number of events per variable in logistic regression analysis. Journal of Clinical Epidemiology, v. 49, n. 12, p. 1373-1379, 1996. http://dx.doi.org/10.1016/ S0895-4356(96)00236-3

PENROSE, E. T. A teoria do crescimento da firma. Campinas: Editora da Unicamp, 2006.

PREARO, L. C. O uso de técnicas estatísticas multivariadas em dissertações e teses sobre o comportamento do consumidor: um estudo exploratório. 2008. Dissertação (Mestrado em Administração)-Faculdade de Economia, Administração e Contabilidade, Universidade de São Paulo, São Paulo, 2008.

PREVEZER, M. The dynamics of industrial clustering in biotechnology. Small Business Economics, v. 9, n. 3, p. 255-271, 1997. http://dx.doi. org/10.1023/A:1017995006003

RAGIN, C.C. Constructing Social Research. Thousand Oaks: Pine Forge, 1994.

RUMELT, R. P. Toward a strategic theory of the firm. In: LAMB, R. B. (Ed.). Competitive Strategic Management. Englewood Cliffs: Prentice-Hall, p. 557-570, 1984.

SHARMA, S. Applied multivariates techniques. New York: Wiley, 1996.

SORENSEN, J; SORENSON, O. From conception to birth: opportunity perception and resource mobilization in entrepreneurship. Advances in Strategic Management, v. 20, p. 71-99, 2003.

STINCHCOMBE, A. L. Organizations and social structure. In: MARCH, J. G. (Ed.). Handbook of Organizations. Chicago: Rand-McNally, 1965. p. 142-193.

STEINDL, J. Small and Big Business: Economic Problem of the size of firms. Oxford: Basil Blackwell, 1945.

THORNHILL, S.; AMIT, R.. Learning about failure: bankruptcy, firm age, and the resource-based view. Organization Science, v. 14, n. 5, p. 497-509, 2003. http://dx.doi.org/10.1287/orsc.14.5.497.16761

VAN WISSEN, L. J. G. Demography of the firm: a useful metaphor? European Journal of Population, v. 18, n. 3, p. 263-279, 2002. http://dx.doi. org/10.1023/A:1019750727018

WEBER, A. Theory of Location of Industries. Chicago: University of Chicago Press, 1957.

WENNBERG, K.; LINDQVIST, G. The effect of clusters on the survival and performance of new firms. Small 
Business Economics, v. 34, n. 3, p. 221-241, 2010. http://dx.doi.org/10.1007/s11187-008-9123-0

WERNERFELT, B. A resource-based view of the firm. Strategic Management Journal, v. 5, n. 2, p. 171-180, 1984. http://dx.doi.org/10.1002/ smj.4250050207

WHOLEY, D. R.; CHRISTIANSON, J. B.; SANCHEZ, S. M. Organization size and failure among health maintenance organizations. American Sociological
Review, v. 57, n. 6, p. 829-842, 1992. http://dx.doi. org/10.2307/2096126

WINTER, S. G. Knowledge and competence as strategic assets. In: TEECE, D. J. (Ed.). The Competitive Challenge. Cambridge: Ballinger, 1987. p. 159-184.

ZAHRA, S. A.; GEORGE, G. Absorptive capacity: a review, reconceptualization, and extension. Academy of Management Review, v. 27, n. 2, p. 185-203, 2002. 